\title{
Mobile Application Development Based on Building Information Modeling for Chinese Users
}

\author{
Huang Zhuohua, Ma Encheng \\ Institute of Building Engineering Software \\ CABR Tech Co., LTD \\ Beijing 100013, China \\ huangzhuohua@cabrtech.com, maencheng@cabrtech.com
}

\begin{abstract}
In China, the mobile devices, especially smart phones and tablet PCs have been growing up with an astonishing speed. Meanwhile Building Information Modeling (BIM) has been one of the most promising developments in the architecture, engineering, and construction (AEC) industries. Since there are very few researches and products on the BIM mobile applications developments in China, based on the author's study and development results, this paper presents a systematic solution on BIM mobile application developments and discusses some critical issues for solving the existed problems, such as the network-speed limitations, simplifications and transferring of the BIM models, as well as platform independence issues to develop well suited mobile applications based on BIM for Chinese users in the AEC. The solutions have been tested in a prototype system.
\end{abstract}

Keywords-Mobile; BIM; AEC; Building Design; Software

\section{INTRODUCTION}

Building Information Modeling (BIM) is one of the most promising developments in the architecture, engineering, and construction (AEC) industries [1]. BIM is a process involving the generation and management of digital representations of physical and functional characteristics of a facility. The resulting building information models become shared knowledge resources to support decision-making about a facility from the earliest conceptual stages, through design and construction, through its operational life and eventual demolition.

Just a few years ago, the constructors in China thought that tablet PC was too expensive to be used for viewing building models in the fields. But now smart phone and tablet PC has become more and more popular in China, so that the architects and engineers have strong willing to use them for each aspect in their daily life. It is a great opportunity to develop mobile applications based on BIM. However, the traditional approach to building design application is not suitable for mobile devices because of the limited memory and connection bandwidth. This paper presents new solutions based on BIM for the architects and engineers in China for their mobile application.

Nawaz, Sarfraz presented a vision where smart-phones and tablet devices carried by construction workers are used to capture the interaction and communication between workers in the field [2]. Dagtas, Serhan presented the framework that enables business applications connected to
SAP through common mobile devices [3]. Larsen, B.D presented a new user friendly technology for accessing large 3D BIMs on mobile devices [4]. The well known commercial software is Autodesk Buzzsaw Mobile, GraphiSoft BIMx for Mobile Devices, Bentley Structural View and Tekla BIMsight Note. But there is still very few related work in China. Since China has her own design codes, and the network speed is still low, it is necessary to study and to develop mobile applications based on Building Information Modeling for Chinese architects and engineers in AEC.

The solutions on network speed limitations, simplifications and transferring of models, and platform independence issues to develop well suited mobile applications based on BIM for Chinese users in AEC are presented in this paper.

\section{PROBlEMS TO BE SOLVED IN DEVELOPING BIM BASED MOBILE APPLICATIONS}

There was an investigation this year which shows that the Chinese network speed is the 90th country among the world as Fig. 1 shown. And the network charges a lot more than other countries [5]. There is still very less wireless hotspot in the urban areas. By using the $3 \mathrm{G}$ network, mobile devices can only reach a very slow speed. A building model should be transferred in a proper way to provide a better experience for the users, so that the following problems should be solved:

(1) For mobile applications, a building model should not be as big as the model for PC in order to get faster loading and viewing speed.

(2) The model should not be kept changing until the operation from a single user is finished, so that different users may view or edit the same model at the same time.

(3) Text, picture, sound and video information from the tablet PC or smart phone should be sent back to the engineer.

(4) The applications should be developed for IOS, Andriod, Windows Phone and other platforms. It is necessary to find a better solution for platform-dependency problems.

\section{SOLUTIONS TO THE PROBLEM SOLVING}

Based on the study and analysis of the problems listed in section II of this paper, and the authors' previous work experience, the solutions have been tested in a prototype system. 


\section{A. Light Weight Building Information Model}

The Building Information Model consists of the information from each phase of the building life cycle, it does not fit the mobile application well. In order to view the Building Information Model in the mobile devices, it is necessary to generate a lightweight model.

For mobile application users, they usually focus on one aspect of the model at each time. The BIM Data Center should have the ability to export sub-model for the owners, the architects, the structural engineers, the MEP engineers or the constructors. Each user can log onto the system and join a project as an owner, an architect or other identity, and then the sub-model for current identity can be viewed and other sub-models will not be loaded.

For large-scale building models, mobile devices do not necessarily need all the elements and attributes. To get the best practice results, the volume of a model should be limited to a certain value, for e.g., 50 Mega Bytes. For some buildings that the same model will be accessed by the most levels, should be recorded only once in the model. If the levels are almost the same, the difference should be recorded separately. And for the attributes, geometric and material attributes should be kept. The others, like the timeline, the current editor, the version and so on should not be in the lightweight model since the mobile application users would not focus on this information.

\section{B. Partial Model TransferIdentify the Headings}

In China, network speed is always a bothering problem. It is critical to divide the whole model into small pieces and transfer them whenever they are needed. The model will be divided into small pieces and whenever the average network speed is detected, they will be combined with the proper partial model. Then they can be downloaded from the server to mobile devices whenever they are within the user's view. The other partial models will be marked in dotted line until they are downloaded.

Restricted by the screen size, very detail information is meaningless while the users are viewing the whole model. The material attributes and the other elements that may not be seen will not be downloaded when the user's viewpoint is outside the building.

\section{Mobile Collaboration}

In our prototype system, the owners and the constructors can point out the errors or the parts which are not as same as they expected. Engineers can also share the ideas though the mobile applications.

Texts, voices and videos can be added by mobile function directly and then sent back as an attachment of the project. Pen tools of the application draw lines and shapes on the current view, the users can use the snapshot function to record them together with the model which can be sent back as an image then.

Text and images can also be bonded with the elements. Whenever the users make the selection of the elements, the application will record the elements index numbers as a list. The users can add text or image-notice for such a selection.
When the engineers get the selection-notification, they will find the elements which should be corrected easily.

\section{Platform independence issues}

Since the applications will be developed for each platform as IOS, Andriod, Windows Phone and more other platforms to satisfy the extensive requirements. It is necessary to find a better solution for platform-dependency problems. Based on the analysis and comparison of the possible solutions, we find that QT and Rhodes are not the best answer. The further studies to find a better solution to platform independence issues will be presented in our next paper.

\section{SYSTEM ARCHITECTURE}

The system architecture is designed as shown in Fig. 2. The data server is usually deployed in a design institute or company; the complete building information model is stored in the central sever of a design institute or company. The partial model is created from the complete model and stored in the web server. The Users can also upload the partial model to the cloud. Mobile devices can read the data directly from the web server or through the cloud. But the feedback can only be sent back to the web server then went back to the data server. The data server receives all changes from the designers and distributes all kinds of feedback to the specific designers.

The mobile application architecture is designed as shown in Fig. 3.

\section{Key Points of The System DeVELOPMENT}

The following points are critical in the development of the system:

A. The collaboration between architects, structural engineers, MEP engineers, owners and constructors is based on the data structure and data organization.

The data server contains all information in the building life cycle; so that each element has its own "4-Dimesonal" information includes the graphical data, physical attributes data, the timeline and the relationship with other elements that are even not in the same professional area.

Whenever a feedback was sent from the mobile application, the web server should call back the datacenter, and then the data maintenance module will be noticed that a change was made. After that the data maintenance module would find the relevant element from other professional area. Finally each related owners, engineers and constructors would be notified with a message which indicates that they should be focused on the recent feedback from a mobile application.

The data organization is currently a prototype that only deals with simple relationships. But the advanced mechanism is being developed that each expert could customize his own collaboration method through an XML file which all regulations are self-defined. 


\section{B. Understanding between the users and the software developers}

The lightweight building model and the feedback method are based on the understanding of the requirements of the owners and the constructors. Since the requirements are complicated and hard to be understood by the software engineer, the software engineering and requirement analysis method are used to make the requirements clear. Also the AEC engineers and software engineers were sitting together each week to correct the mathematical model and the data format. The process was held during the data server development so that the mobile application could meet the experts' requirements.

\section{CONCLUSIONS}

To meet the requirements of mobile applications based on BIM for Chinese AEC users; this paper studied the solutions on the network speed and collaboration problems, model simplifications and transferring methods, as well as platform independence issues. The study results can be concluded as follows:

(1) In order to view the Building Information Model in the mobile devices, it is necessary to generate a lightweight model.

(2) To get the best practice results, the volume of a model should be limited to a certain value, for e.g. 50 Mega Bytes.

(3) The model should be divided into small pieces and whenever the average network speed is detected, they will be combined with the proper partial model.
(4) It is necessary to find a better solution for platformdependency problems. Based on the analysis and comparison of the possible solutions, we find that QT and Rhodes are not the best answer.

The authors hope that more researches can be made in this area to truly push forward the Chinese BIM process.

\section{ACKNOWLEDGMENT}

The authors would like to thank the project fund from CABR Tech Co., LTD for their supports to this study.

\section{REFERENCES}

[1] Chuck Eastman, Paul Teicholz, Rarael Sacks, Kathleen Liston BIM Handbook: A Guide to Building Information Modeling for owners, managers, designers, engineers, and Contractors, Second edition, p1, ISBN: 978-0-470-54137-1

[2] Nawaz, Sarfraz; Efstratiou, Christos; Mascolo, Cecilia; Soga, Kenichi MCSS'12 - Proceedings of the 1st ACM Workshop on Mobile Systems for Computational Social Science, p 28-32, 2012, ISBN-13: 9781450313247.

[3] Dagtas, Serhan; Natchetoi, Yuri; Wu, Huaigu; Hamdi, Louenas 7th IEEE/IFIP Working Conference on Software Architecture, WICSA 2008, p 41-50, 2008, ISBN-10: 0769530923, ISBN-13: 9780769530925.

[4] Larsen, B.D.1 eWork and eBusiness in Architecture, Engineering and Construction - Proceedings of the European Conference on Product and Process Modelling 2012, ECPPM 2012, p 505-508.

[5] http://www.cnr.cn/gundong/201202/t20120204_509123212.shtml

[6] Sarah Allen, Vidal Graupera, Lee LundriganPro Smartphone CrossPlatform Development: iPhone, BlackBerry, Windows Mobile, and Android Development and Distribution, EISBN: 978-1-4302-2868-4

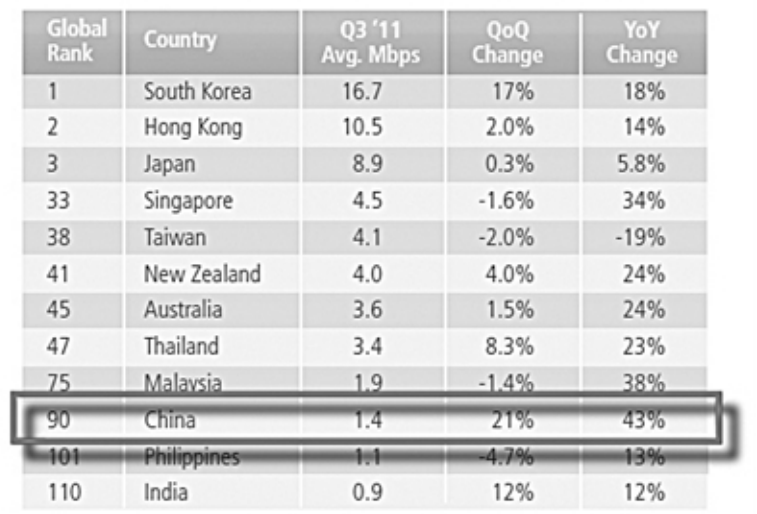

Figure 1. 


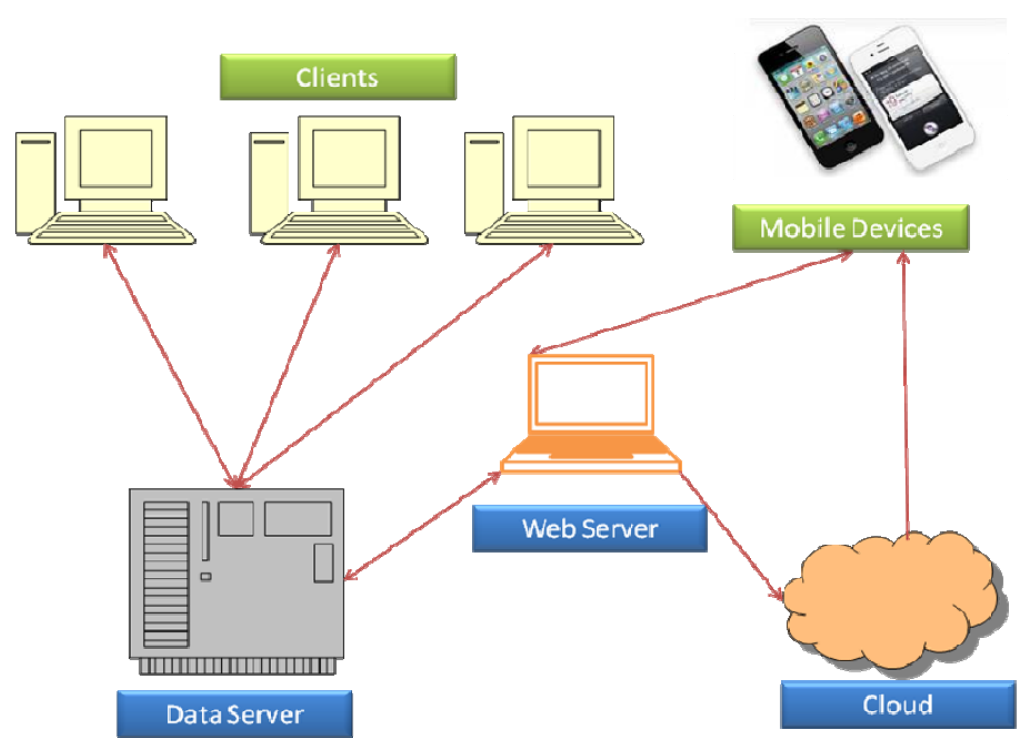

Figure 2. System architecture

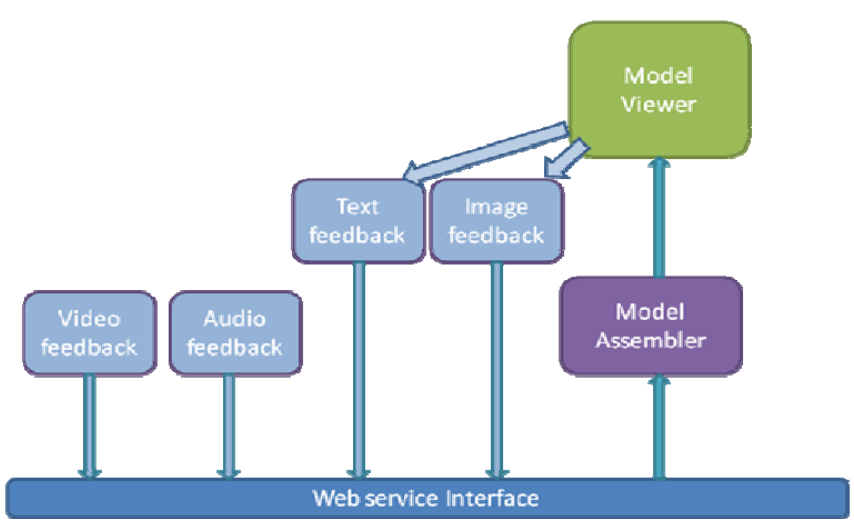

Figure 3. Mobile application architecture 